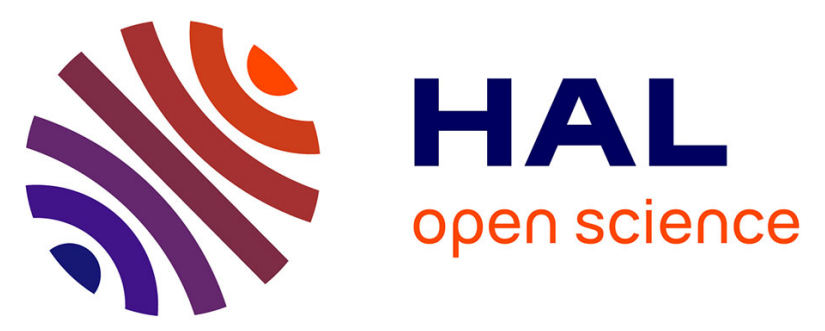

\title{
SHAMAN: a user-friendly website for metataxonomic analysis from raw reads to statistical analysis
}

Stevenn Volant, Pierre Lechat, Perrine Woringer, Laurence Motreff, Pascal Campagne, Christophe Malabat, Sean Kennedy, Amine Ghozlane

\section{To cite this version:}

Stevenn Volant, Pierre Lechat, Perrine Woringer, Laurence Motreff, Pascal Campagne, et al.. SHAMAN: a user-friendly website for metataxonomic analysis from raw reads to statistical analysis. BMC Bioinformatics, 2020, 21 (1), pp.345. 10.1186/s12859-020-03666-4 pasteur-02951458

\section{HAL Id: pasteur-02951458}

\section{https://hal-pasteur.archives-ouvertes.fr/pasteur-02951458}

Submitted on 28 Sep 2020

HAL is a multi-disciplinary open access archive for the deposit and dissemination of scientific research documents, whether they are published or not. The documents may come from teaching and research institutions in France or abroad, or from public or private research centers.
L'archive ouverte pluridisciplinaire HAL, est destinée au dépôt et à la diffusion de documents scientifiques de niveau recherche, publiés ou non, émanant des établissements d'enseignement et de recherche français ou étrangers, des laboratoires publics ou privés.

\section{(c)(1)}

Distributed under a Creative Commons Attribution| 4.0 International License 


\title{
SHAMAN: a user-friendly website for metataxonomic analysis from raw reads to statistical analysis
}

\author{
Stevenn Volant ${ }^{1}$, Pierre Lechat ${ }^{1}$, Perrine Woringer ${ }^{1}$, Laurence Motreff ${ }^{2}$, Pascal Campagne ${ }^{1}$, \\ Christophe Malabat ${ }^{1}$, Sean Kennedy ${ }^{2}$ and Amine Ghozlane $e^{1,2^{*}}$
}

\begin{abstract}
${ }^{*}$ Correspondence:
amine.ghozlane@pasteur.fr

${ }^{1}$ Hub de Bioinformatique et Biostatistique - Département Biologie Computationnelle, Institut Pasteur, USR 3756 CNRS, 28 Rue Du Docteur Roux, 75015 Paris, France ${ }^{2}$ Biomics - Département Génomes et Génétique, Institut Pasteur, 28 Rue du Docteur Roux, 75015 Paris, France
\end{abstract}

\begin{abstract}
Background: Comparing the composition of microbial communities among groups of interest (e.g., patients vs healthy individuals) is a central aspect in microbiome research. It typically involves sequencing, data processing, statistical analysis and graphical display. Such an analysis is normally obtained by using a set of different applications that require specific expertise for installation, data processing and in some cases, programming skills.

Results: Here, we present SHAMAN, an interactive web application we developed in order to facilitate the use of (i) a bioinformatic workflow for metataxonomic analysis, (ii) a reliable statistical modelling and (iii) to provide the largest panel of interactive visualizations among the applications that are currently available. SHAMAN is specifically designed for non-expert users. A strong benefit is to use an integrated version of the different analytic steps underlying a proper metagenomic analysis. The application is freely accessible at http://shaman.pasteur.fr/, and may also work as a standalone application with a Docker container (aghozlane/shaman), conda and R. The source code is written in R and is available at https://github.com/aghozlane/shaman. Using two different datasets (a mock community sequencing and a published 16S rRNA metagenomic data), we illustrate the strengths of SHAMAN in quickly performing a complete metataxonomic analysis.
\end{abstract}

Conclusions: With SHAMAN, we aim at providing the scientific community with a platform that simplifies reproducible quantitative analysis of metagenomic data.

Keywords: Metagenomics, Differential analysis, Visualization, Web application

\section{Background}

Quantitative metagenomic techniques have been broadly deployed to identify associations between microbiome and environmental or individual factors (e.g., disease, geographical origin, etc.). Analyzing changes in the composition and/or in the abundance of microbial communities yielded promising biomarkers, notably associated with liver

(c) The Author(s). 2020 Open Access This article is licensed under a Creative Commons Attribution 4.0 International License which permits use, sharing, adaptation, distribution and reproduction in any medium or format, as long as you give appropriate credit to the original author(s) and the source, provide a link to the Creative Commons licence, and indicate if changes were made. The images or other third party material in this article are included in the article's Creative Commons licence, unless indicated otherwise in a credit line to the material. If material is not included in the article's Creative Commons licence and your intended use is not permitted by statutory regulation or exceeds the permitted use, you will need to obtain permission directly from the copyright holder. To view a copy of this licence, visit http://creativecommons.org/licenses/by/4.0/. The Creative Commons Public Domain Dedication waiver (http://creativecommons.org/publicdomain/zero/1.0/) applies to the data made available in this article, unless otherwise stated in a credit line to the data. 
cirrhosis [1], diarrhea [2], colorectal cancer [3], or associated with various pathogenic [4] or probiotic effects [5] on the host.

In metataxonomic studies, a choice is made prior to sequencing in order to specifically amplify one or several regions of the rRNA (usually the $16 \mathrm{~S}$ or the $18 \mathrm{~S}$ rRNA genes for procaryotes/archaea and the ITS, the $23 \mathrm{~S}$ or the $28 \mathrm{~S}$ rRNA gene for eukaryotes) so that the composition of microbial communities may be characterized with affordable techniques.

A typical workflow includes successive steps: (i) OTU (Operational Taxonomic Unit) picking (dereplication, denoising, chimera filtering and clustering) [6], (ii) OTU quantification in each sample and (iii) OTU annotating with respect to a reference taxonomic database. This process may require substantial computational resources depending on both the number of samples involved and the sequencing depth. Several methods are currently available to complete these tasks, such as Mothur [7], Usearch [8], DADA2 [9] or Vsearch [10]. The popular application Qiime [11] simplifies these tasks (i to iii) and visualizations by providing a python-integrated environment. Schematically, once data processing is over, both a contingency table and a taxonomic table are obtained. They contain the abundance of OTUs in the different samples and the taxonomic annotations of OTUs, respectively. The data are normally represented in the standard BIOM format [12].

Statistical analysis is then performed to screen significant variation in microbial abundance. To this purpose, several $R$ packages were developed, such as Metastats [13] or Metagenomeseq [14]. It is worth noticing that other approaches which were originally designed for RNA-seq, namely DESeq2 [15] and EdgeR [16], are also commonly used to carry out metataxonomic studies $[17,18]$. They provide an $\mathrm{R}$ integrated environment for statistical modelling in order to test the effects of a particular factor on OTU abundance. Nevertheless using all of these different methods requires technical skills in Unix, $\mathrm{R}$ and experience in processing metagenomics data. To this end, we developed SHAMAN in order to simplify the analysis of metataxonomic data, especially for users who are not familiar with the technicalities of bioinformatic and statistical methods that are commonly applied in this field.

SHAMAN is an all-inclusive approach to estimate the composition and abundance of OTUs, based on raw sequencing data, and to perform statistical analysis of processed files. First, the user can submit raw data in FASTQ format and define the parameters of the bioinformatic workflow. The output returns, a BIOM file for each database used as a reference for annotation, a phylogenetic tree in Newick format as well as FASTA-formatted sequences of all OTUs that were identified. The second step consists in performing statistical analysis. The user has to provide a "target" file that associates each sample with one or several explanatory variables. These variables are automatically detected in the target file. An automatic filtering of the contingency matrix of OTUs may be activated in order to remove features with low frequency. Setting up the contrasts to be compared is also greatly simplified. It consists in filling in a form that orients the choices of users when defining the groups of interest. Several options to visualize data are available at three important steps of the process: quality control, bio-analysis and contrast comparison. At each step, a number of common visual displays are implemented in SHAMAN to explore data. In addition, SHAMAN also includes a variety of original displays that is not available in other applications such as an abundance tree to visualize count distribution according to the taxonomic tree and variables, or the logit plot to compare feature $p$-values in two 
contrasts. Figures may be tuned to emphasize particular statistical results (e.g., displaying significant features in a given contrast only, displaying intersections between contrasts), to be more specific (e.g. feature abundance in a given modality) or to improve the aesthetics of the graph (by changing visual parameters). Figures fit publication standards and the corresponding files can be easily downloaded.

Several web applications were developed to analyze data of metataxonomic studies, notably, FROGS [19], ASaiM [20], Qiita [21] as well as MetaDEGalaxy [22] for bioinformatic data processing, Shiny-phyloseq [23] for statistical analysis, Metaviz [24] and VAMPS2 [25] that make a particular focus on data visualization. While these interfaces propose related functionalities, the main specificity of SHAMAN is to combine of all these steps in a single user-friendly application. Last, SHAMAN may keep track of a complete analysis which may be of particular interest for matters of reproducibility.

\section{Material and method}

SHAMAN is implemented in $\mathrm{R}$ using the shiny-dashboard framework. The application is divided into three main components (Fig. 1): a bioinformatic workflow to process the raw FASTQ-formatted sequences, a statistical workflow to normalize and further analyse data, as well as a visualization platform. Users may run each component of the workflow independently or run the whole process from raw FASTQ data to visualization. SHAMAN provides, for each component, scores and figures that summarize the quality of data processing. When installed with Docker, SHAMAN has low computing resource requirements with a minimum $1 \mathrm{Ghz}$ processor, $1 \mathrm{Gbyte}$ of ram memory and 3.4Gbytes of disk.

\section{Bioinformatic workflow in SHAMAN}

The metataxonomic pipeline implemented in SHAMAN relies on the Galaxy platform [26]. All calculations are remotely done on galaxy.pasteur.fr; this process is transparent to the user. The pipeline flows in the following manner:

1 Optional filtering of reads. It is worth noticing that previous studies, e.g. carried out on mosquito microbiota [27], showed that some non-annotated OTUs turned to be sequences of the host organism. To overcome such issues, the user can optionally filter out reads that align with the host genome and the PhiX174 genome

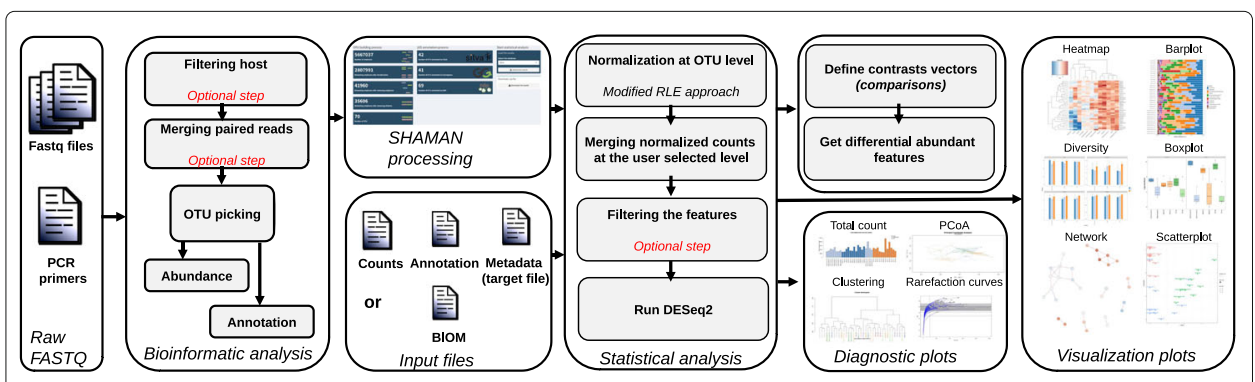

Fig. 1 SHAMAN workflow. SHAMAN can start from raw reads or from processed data. In this last case, it needs at least three tables, the count matrix, the annotation table and the metadata which can either be provided into three different files or by using the BIOM format. The user can then select the variables of interest and add some batch effects. Then, contrast vectors can easily be defined and interactive visualizations are available 
(used as a control in Illumina sequencers). The latter task is performed with Bowtie2 v2.2.6 [28].

2 Quality of reads is checked with AlienTrimmer [29] v0.4.0, a software for trimming off contaminant sequences and clipping.

3 Paired-end reads are then merged with Pear [30] v0.9.10.1.

4 OTU picking, taxonomic annotation and OTU quantification are performed using Vsearch [10] v2.3.4.0, a software that was shown to be both accurate and efficient $[6,31]$. The OTU picking process consists in five steps, i.e., dereplication, singleton removal, chimera detection, clustering and alignment. It follows the approach and default parameters previously described by the Uparse pipeline [8]. Input amplicons are aligned against the set of detected OTUs to create a contingency table that contains the number of amplicons assigned to each OTU. This step aims at refining OTU counts by including singletons that correspond to sequences with a reasonable amount of sequencing errors (i.e., $<3 \%$ ).

5 The taxonomic annotation of OTUs is performed based on various databases, i.e., with SILVA [32] rev. 132 SSU (for 16S, 18S rRNA genes) and LSU (for $23 \mathrm{~S}$ and $28 \mathrm{~S}$ rRNA genes), Greengenes [33] (for 16S, 18S rRNA genes) and Underhill rev. 1.6.1 [34], Unite rev. 8.0 [35] and Findley [36] for ITS rRNA sequences. These databases are kept up-to-date every two month with biomaj.pasteur.fr.

6 OTU annotations are filtered according to their identity with the reference [37]. Phylum annotations are kept when the identity between the OTU sequence and the reference sequence is $\geq 75 \%, \geq 78.5 \%$ for classes, $\geq 82 \%$ for orders, $\geq 86.5 \%$ for families, $\geq 94.5 \%$ for genera and $\geq 98 \%$ for species. In addition, a taxonomic inference based on a naive Bayesian approach, RDP classifier [38] v2.12, is systematically provided. By default, RDP annotations are included whenever the annotation probability is $\geq 0.5$. All the above-mentioned thresholds may be tuned by the user.

7 A phylogenetic analysis of OTUs is provided: multiple alignments are obtained with Mafft [39] v7.273.1, filtering of regions that are insufficiently conserved is made using BMGE [40] v1.12 and finally, FastTree [41] v2.1.9 is used to infer the phylogenetic tree. Based on the latter tree, a Unifrac distance [42] may be computed in SHAMAN to compare microbial communities.

The outcomes of the overall workflow are stored in several files: a BIOM file (per reference database), a phylogenetic tree as well as a summary file specifying the number of elements passing the different steps of the workflow. The data are associated to a key that is unique to a project. Such a key allows to automatically re-load all results previously obtained within a given project.

\section{Statistical workflow in SHAMAN}

The statistical analysis in SHAMAN is based on DESeq2 which is a method to model OTU counts with a negative binomial distribution. It is known as one of the most accurate approach to detect differentially abundant bacteria in metagenomic data $[17,18]$. Relying on robust estimates of variation in OTUs, the DESeq2 method has suitable performances even with datasets characterized by a relatively low number of observations per group (together with a high number of OTUs). 
This method typically requires the following input files: a contingency table, a taxonomic table and a target file describing the experimental design. These data are processed to generate a meta-table that assign to each OTU, a taxonomic annotation and a raw count per sample.

\section{Normalization}

Normalization of the raw counts is one of the key issues when analyzing microbiome experiments. The uniformity of the sequencing depth is affected by sample preparation and dye effects [43]. Normalizing data is therefore expected to increase the accuracy of comparisons. It is done by adjusting the abundance of OTUs across samples. Four different normalization methods are currently implemented in SHAMAN. For the sake of consistency, all of these methods are applied at the OTU level.

A first method is the relative log expression (RLE) normalization and is implemented in the DESeq2 package. It consists in calculating a size factor for each sample, i.e., a multiplication factor that increases or decreases the OTU counts in samples. It is defined as the median ratio between a given count and the geometric mean of the corresponding OTU. Such a normalization was shown to be suited for metataxonomic studies [17]. In practice, many OTUs are found in a few samples only, which translate into sparse count matrices [14]. In this case, the RLE method may lead to a defective normalization - as only a few OTU are taken into account - or might be impossible if all OTUs have a null abundance in one sample at least. In the R package Phyloseq [44], the decision was made to calculate a modified geometric mean by taking the $n$-th root of the product of the non-zero counts, which is equivalent to replacing the null abundance by a count of 1 . This approach might impact the normalization process when the input matrix is very sparse. As a consequence, we decided to include two new normalization methods : the non-null and the weighted non-null normalizations. They are modified versions of the original RLE so that they better account for matrix sparsity (number of elements with null values divided by the total number of elements). In the non-null normalization (1) cells with null values are excluded from the computation of the geometric mean. This method therefore takes all OTUs into account when estimating the size factor. In the second method that we coined the weighted non-null normalization (2), weights are introduced so that OTUs with a larger number of occurrences have a higher influence when calculating the geometric mean.

Assume that $C=\left(c_{i j}\right)_{1 \leq i \leq k ; 1 \leq j \leq n}$ is a contingency table where $k$ and $n$ are the number of features (e.g. OTUs) and the number of samples, respectively. Here, $c_{i j}$ represents the abundance of the feature $i$ in the sample $j$. The size factor of sample $j$ is denoted by $s_{j}$.

$$
\begin{aligned}
& s_{j}^{(1)}=\operatorname{median}_{i} \frac{c_{i j}}{\left(\prod_{k \in S_{i}} c_{i k}\right)^{1 / n_{i}}}, \\
& s_{j}^{(2)}=\text { w.median }_{i} \frac{c_{i j}}{\left(\prod_{k \in S_{i}} c_{i k}\right)^{1 / n_{i}}},
\end{aligned}
$$

where $S_{i}$ stands for the subset of samples with non null values for the feature $j$ and $n_{i}$ is the size of this subset. The function w.median corresponds to a weighted median.

An alternative normalization technique is the total counts [45] which is convenient for highly unbalanced OTU distribution across samples. 
Using a simulation-based approach, we addressed the question of the performance of the non-null and the weighted non-null normalization techniques when the matrix sparsity and the number of observations increase. We compared these new methods to those normally performed with DESeq2 and Phyloseq. To do so, we normalized 500 simulated matrices using the function makeExampleDESeqDataSet of DESeq2 with varied sparsity levels (i.e., $0.28,0.64$ and 0.82 ) and different numbers of observations (i.e., $n=4,10$ and 30). We calculated the average coefficient of variation (CVmean) [46] for each normalization method (Fig. S1). Considering that these OTUs are assumed to have relatively constant abundance within the simulations, the coefficient of variation is expected to be lower when the normalization is more efficient. In this simulation-based comparison, the non-null and the weighted non-null normalization methods exhibited a lower coefficient of variation as compared to the other methods, when sparsity in the count matrix is high and the number of observations is increased. These differences were clear especially when comparing the normalization methods used in DESeq2 and Phyloseq to the weighted non-null normalization (sparsity ratio of $0.28,0.64$ and 0.82 , with 30 samples; t-tests $p<0.001$ ) (Fig. S1).

\section{Contingency table filtering}

In metataxonomic studies, contingency tables are often very sparse and after statistical analysis, some differences associated with $p$-values $<0.05$ are not necessarily of great relevance, due to violated assumptions. This may arise when a feature, distributed in many samples with a low abundance, is slightly more abundant in one group of comparison. These artifacts are generally excluded by DESeq2 with an independent filtering. Furthermore, if a feature is found in high abundance in a few samples only (and count is 0 in the other samples), it may lead to non-reliable results. Such distributions may also affect the normalization process as well as the dispersion estimates. In order to avoid misinterpretation of results, we propose an optional extra-step of filtering, by excluding features characterized by a low abundance and/or a low number of occurrence in samples (e.g. features occurring in less than $20 \%$ of the samples). To set a by-default abundance threshold, SHAMAN searches for an inflection point at which the curve between the number of observations and the abundance of features changes from being linear to concave. This process is performed with linear regression in the following manner:

1 We define $I$ the interval $\left[\min _{j}\left(\sum_{i} c_{i j}\right) ; \frac{\sum_{i j} c_{i j}}{k}\right]$.

2 For each $x \in I$, we compute $h(x)$ defined as the number of observations with a total abundance higher than $x$.

3 We compute the linear regression between $h(x)$ and $x$.

4 The intercept is set as the default threshold.

(see Appendix 1 for more information). This extra-filtering is more stringent than the DESeq2 process and normally results in decreased computation time. The impact of filtering steps may be visually assessed with plots displaying features that will be included in the analysis and those that will be discarded.

\section{Statistical modelling}

The statistical model relies on the variables that are loaded in the file of experimental design. By default, all variables are included in the model but the end-user can edit this 
selection and further add interactions between variables of interest. In addition, other variables such as batches or clinical data (e.g., age, sex, etc.) may be used as covariates. SHAMAN then automatically checks whether the model is statistically suitable (i.e., whether the parameters may be estimated properly). When it is not the case, a warning message appears and a "how to" box proposes a practical way to solve the issue. In SHAMAN, statistical models may be fitted at any taxonomic levels: normalized counts are summed up within a given taxonomic level.

To extract features that exhibit significant differential abundance (between two groups), the user must define a contrast vector. Both a guided mode and an expert mode are available in SHAMAN. In the guided mode, the user specifies the groups to be compared using a dropdown menu. This mode is only available for DESeq2 v1.6.3 which is implemented in the DESeq2shaman package (https://github.com/aghozlane/DESeq2shaman). In advanced comparisons, the user may define a contrast vector by specifying coefficients (e.g., - $1,0,1)$ assigned to each variable.

\section{Visualization in SHAMAN}

After running a statistical analysis, many displays are available:

(i) Diagnostic plots (such as barplots, boxplots, PCA, PCoA, NMDS and hierarchical clustering) help the user examine both raw and normalized data. For instance, these plots may reveal clusters, sample mislabelling and/or batch effects. Scatterplots of size factors and dispersion estimates (i.e., estimates that are specific to DESeq2) are useful when assessing both the relevance and robustness of statistical models. PCA- and PCoA-plots associated with a PERMANOVA test may be used as preliminary results in the differential analysis as they may reveal global effects among groups of interest.

(ii) All significant features are gathered in a table including, the base mean (mean of the normalized counts), the fold change (i.e., the factor by which the average abundance changes from one group to the other), as well as the corresponding adjusted $p$-values. The user may view tables for any contrasts and can export them into several formats. Volcano plots and bar charts of $p$-values and $\log 2$ fold change are also available in this section.

(iii) A global visualization section provides a choice of 9 interactive plots such as barplots, heatmaps and boxplots to represent differences in abundance across groups of interest. Diversity plots display the distribution of various diversity indices: alpha, beta, gamma, Shannon, Simpson and inverse Simpson. Scatterplots and network plots may reveal associations between feature abundance and other variables from the target file. To explore variations of abundance across the taxonomic classification, we included an interactive abundance tree and a Krona plot [47]. Rarefaction curves are of great use to further consider the number of features in samples with respect to the sequencing depth.

(iv) In the comparison section, plots of comparisons among contrasts may be created. Several options are available such as, Venn diagram or upsetR graph [48] (displaying subsets of common features across contrast), heatmap, a logit plot [49] (showing the $\log 2$ fold-change values in each feature), a density plot and a multiple Venn diagram to summarize the number of features captured by each contrast. All these graphs can be exported into four format (eps, png, pdf and svg). 


\section{User case datasets}

To illustrate how SHAMAN operates, we analysed two datasets. The first dataset originates from a mock sequencing we performed on purpose (a standard practice when assessing metagenomics methods). The second dataset is publicly available and originates from a published study (the afribiota dataset, [50]). The latter dataset was collected to perform a typical differential analysis (i.e., a very common approach in metagenomics). In both analyses, we submitted the raw FASTQ files and provided a target file containing sample information (needed for statistical analysis).

\section{Zymo mock dataset}

The mock sequencing (EBI ENA code PRJEB33737) of the ZymoBIOMICS ${ }^{\mathrm{TM}}$ Microbial Community DNA was performed with an Illumina MiSeq resulting in 12 samples of $257,000 \pm 85,000$ (mean $\pm \mathrm{SD}$ ) sequences of 300 -base-long paired-end reads. The composition of the Zymo mock community is known and is composed with 8 phylogenetically distant bacterial strains, 3 of which are gram-negative and 5 of which are gram-positive. DNA of two yeast strains that are normally present in this community were not amplified. Genomic DNA from each bacterial strain was mixed in equimolar proportions (https:// www.zymoresearch.com/zymobiomics-community-standard). We compared the impact of both the number of amplification cycles ( 25 and 30 cycles) and the amount of DNA loaded in the flow cell (0.5ng and $1 \mathrm{ng}$ ), on the observed microbial abundance. Each sample was sequenced 3 times (experimental plan provided in supplementary materials). Sequencing report provided by the sequencing facility indicated the presence of contaminants. To handle this issue, we filtered out the genera occurring in less than 12 samples and outliers with a reduced log abundance as compared to the other genera (Fig. S2).

\section{Afribiota dataset}

The second dataset included 541 samples of microbial communities in stunted children aged 2-5y living in sub-Saharan Africa (EBI ENA code PRJEB27868) [50]. Three groups (nutritional status) of individuals were considered: $\mathrm{NN}=$ non stunted, $\mathrm{MCM}=$ moderately stunted, MCS=severely stunted. samples originated from the small intestine fluids (gastric and duodenal) and feces. The authors performed the bioinformatic treatment with QIIME framework and the statistical analysis with several R packages including Phyloseq for the normalization and DESeq2 for the differential analysis. Using SHAMAN, raw reads were filtered against Human HG38 and PhiX174 genomes. A total of 2386 OTUs were calculated and $76 \%$ were annotated with SILVA database at genus level. The sparsity rate of the contingency table was high with 0.84 . In consequence, we used the weighted non-null normalization which is particularly efficient when the matrix is highly sparse (Fig. S1). Two analyses were performed, a global analysis that included duodenal, gastric as well as feces samples and a more specific analysis including fecal samples only. Statistical models included the following variables, age, gender, country of origin and nutritional status.

\section{Benchmarking}

Last, we compared the running time performance of SHAMAN with five other web applications for metataxonomy studies (ASaiM, FROGS, MetaDEGalaxy and Qiita). For each web interface, we submitted the raw sequencing reads of the Zymo mock dataset and estimated the time to generate a BIOM containing the contingency matrix and OTU annotation. Qiita and MetaDEGalaxy were used remotely, FROGS was installed on 
galaxy.pasteur.fr and ASAIM was installed on Mac book pro with a core i7 6cpu with 32Gbytes of ram.

\section{Results and discussion}

\section{User cases}

Data analysis in SHAMAN may easily be done by users who are not familiar with command-line analyses. In this paper we analyzed two datasets.

In the analysis of the Zymo mock dataset the 8 bacterial strains present in the samples were suitably detected (Fig. 2). We then defined a statistical model to test the effects of the two varying experimental factors: the amount of DNA and the number of amplification cycles and the interaction between th ese variables. The statistical comparison showed a stronger impact of the number of amplification cycles as compared to the amount of DNA. While we found no differential features between $0.5 \mathrm{ng}$ and $1 \mathrm{ng}$ DNA for each group of number of cycle ( 25 and 30 cycles), the comparison of the number of amplification cycles within each group of DNA amount revealed a significant impact on the observed abundance of mock bacteria (Tables S1, S2). These results are in agreement with previous studies that presented the PCR-induced bias on similar mixtures [51, 52].

Regarding the analysis of the second dataset with SHAMAN, overall our results were highly consistent with those of Vonaesch et al. [50]. We detected a significant change in the community composition between gastric and duodenal samples as compared to feces samples at Genus level (Fig. 3a) (PERMANOVA, $\mathrm{P}=0.001$ ). The most abundant genera were reported in Fig. S3. $\alpha$-Diversity was not affected by stunting (Fig. 3b). We looked for a distinct signature of stunting in the feces. In the volcano plot (Fig. 3c), we represent genera with differential abundance between stunt samples as compared to non-stunt (complete list available in Table S3). Twelve microbial taxa, corresponding to members of the oropharyngeal core microbiota, were over-represented in feces samples of stunted children as compared to non-stunted children. More particularly Porphyromonas, Neisseira and Lactobacillus (Fig. 3d) appeared more abundant. All those findings were in

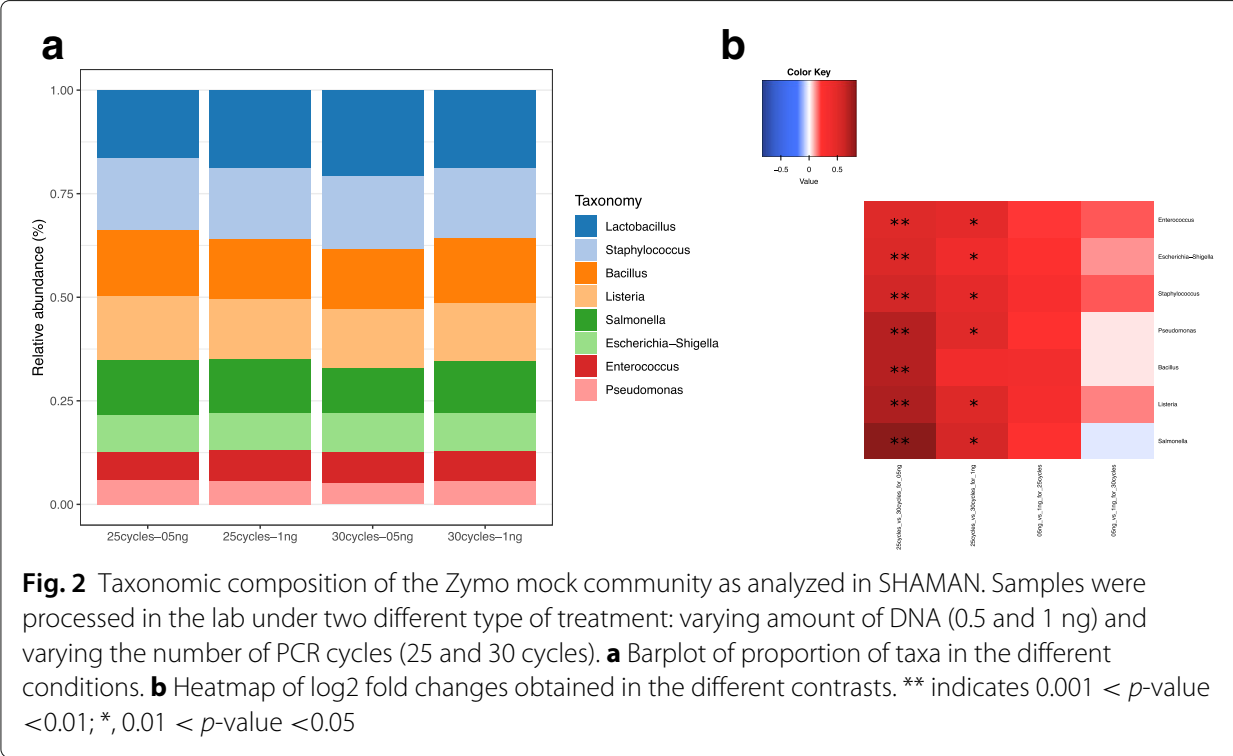




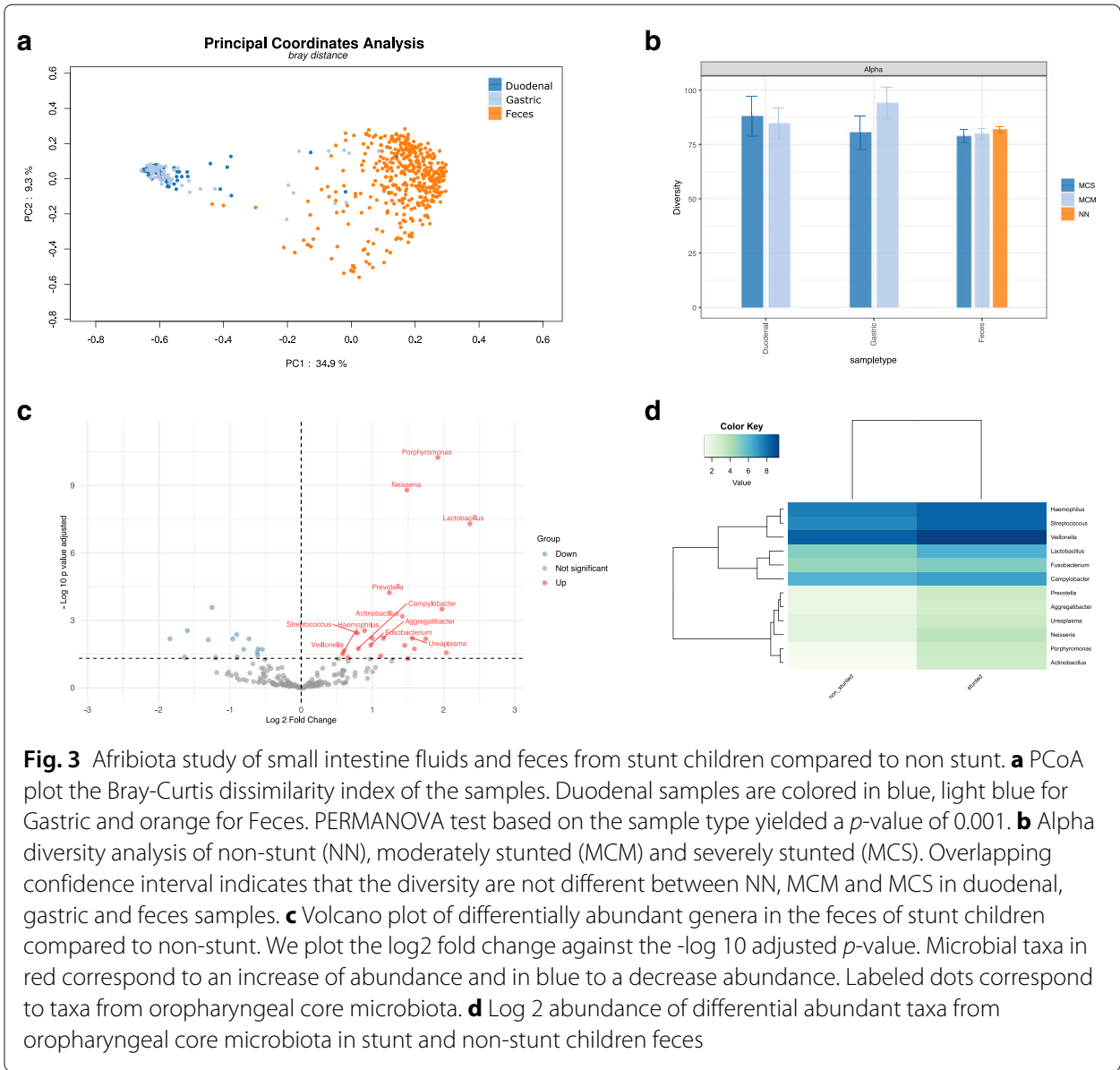

agreement with the conclusions of the Afribiota consortium while being obtained within a few minutes of interaction with the SHAMAN interface.

\section{Mapping SHAMAN among the other existing tools}

To date, several tools have been developed for the analysis of metagenomic data. Relative to these existing tools, SHAMAN presents a number of interesting features and novelties. We made a brief qualitative assessment of the strengths and limits of SHAMAN, in comparison with other web interfaces designed for metataxonomic analyses (see Table 1). We first defined a list of important considerations that have practical implications for the user such as the possibility to process raw sequencing data, the existence of a statistical workflow and/or a visualization platform, possibility to store data and accessibility. For each web interface, we then evaluated whether it met those criteria. We believe that a nested solution, such as SHAMAN, is highly suited for producing robust and reliable results. Any results in SHAMAN may be cross-checked with a quantification or an annotation performed at an earlier stage.

Comparison of computation time revealed that SHAMAN was faster than the other five web application to process the data of Zymo mock dataset. FROGS and QIITA are also convenient solutions for data processing since the whole OTU processing was performed in few hours. In both case, they provided an accurate description of the community as the 8 main communities composing the mock were correctly detected. On 


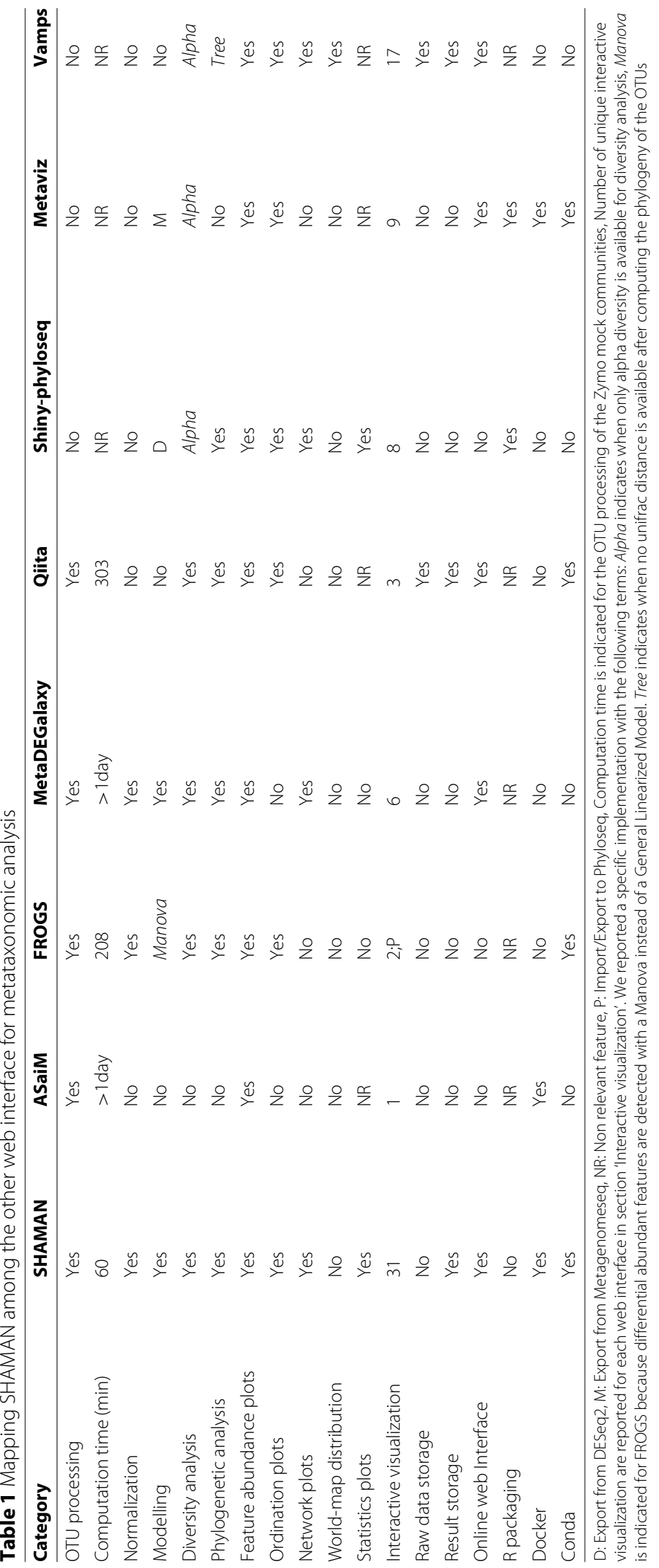


the other side, the computation time obtained with ASaiM and MetaDEGalaxy appeared much longer as we could not obtain a BIOM file after several days of calculation. Results obtained with Frogs and Qiita are reported on figshare (10.6084/m9.figshare.11815860). Furthermore several applications (as presented in Table 1), impose the burden of importing/exporting $\mathrm{R}$ objects which requires skills in $\mathrm{R}$ programming. This may also represent a source of reproducibility issues, notably in terms of compatibility of the packages over time.

\section{Conclusions}

SHAMAN enables users to lead most of the classical metagenomics approaches. It also makes use of statistical analyses to provide support to each data visualization. The possibility to deploy SHAMAN locally constitutes an important feature when the data cannot be submitted on servers for privacy issues or because of insufficient internet access. SHAMAN also simplifies the access to open computational facilities, making a careful use of the dedicated server, galaxy.pasteur.fr.

Currently SHAMAN is limited to metataxonomic analyses. In a close future, we plan to extend our application to whole genome analysis, notably by using of microbial gene catalogs. Several catalogs are currently available to study human, mouse, cow, as well as ocean microbial diversity. A perspective will be to combine these results with metataxonomic data, and to perform integrative analyses.

During the development of SHAMAN, we felt a strong interest of the metagenomics community in our application. We recorded 82 active users per month in 2019 (535 unique visitors in total) and 800 downloads of the docker application. We expect that SHAMAN will help researcher perform a quantitative analysis of metagenomics data.

\section{Availability and requirements}

Project name: SHAMAN

Project home page: http://shaman.pasteur.fr, https://github.com/aghozlane/shaman

Operating system: Platform independent

Programming language: $\mathrm{R}$

Other requirements: Python 3

License: GNU GPL V3

Any restrictions to use by non-academics: No

\section{Supplementary information}

Supplementary information accompanies this paper at https://doi.org/10.1186/s12859-020-03666-4.

Additional file 1: Supplementary materials (Appendix 1, Supplementary Figures S1-S3, Supplementary Tables S1-S2).

Abbreviations

BIOM: Biological observation matrix; CV: Coefficient of variation; DNA: Deoxyribonucleic acid; EBI: European bioinformatic institute; ENA: European nucleotide archive; ITS: Internal transcribed spacer; MCM: Moderately stunted; MCS: Severely stunted; NN: Non stunted; NMDS: Non-metric multidimensional scaling; OTU: Operational taxonomic unit; PCA: Principal component analysis; PCoA: Principal coordinates analysis; PCR: Polymerase chain reaction; PERMANOVA: Permutational analysis of variance; RLE: Relative log expression; rRNA: Ribosomal ribonucleic acid

\section{Acknowledgements}

We thank Hugo Varet for helpful discussions about DESeq2, Fabien Mareuil for the help to deploy SHAMAN computation on Galaxy and Youssef Ghorbal for the maintenance of the databanks, as well as the IT System Department of Institut Pasteur, who manages installation and update of tools on TARS cluster. 


\section{Authors' contributions}

SV,PL, PW, CM and AG implemented SHAMAN; LM and SK performed the mock sequencing; SV, PC and AG wrote the publication. All authors have read and approved the manuscript.

\section{Funding}

This study was supported by the Institut Pasteur.

\section{Availability of data and materials}

Sequence reads of Zymo Mock have been deposited in the European Nucleotide Archive, https://www.ebi.ac.uk/ena/ accession no. PRJEB33737. The datasets generated, analysed during the current study and the simulation script of the supplementary figure 2 are available in the repository: 10.6084/m9.figshare.11815860.

Ethics approval and consent to participate
No ethics approval was required for the study.

\section{Consent for publication}

Not applicable.

\section{Competing interests}

The authors declare that they have no competing interests.

Received: 13 February 2020 Accepted: 16 July 2020

Published online: 10 August 2020

\section{References}

1. Qin N, Yang F, Li A, Prifti E, Chen Y, Shao L, Guo J, Le Chatelier E, Yao J, Wu L, Zhou J, Ni S, Liu L, Pons N, Batto JM, Kennedy SP, Leonard P, Yuan C, Ding W, Chen Y, Hu X, Zheng B, Qian G, Xu W, Ehrlich SD, Zheng S, Li L. Alterations of the human gut microbiome in liver cirrhosis. Nature. 2014;513:59-64. https://doi.org/10.1038/ nature 13568

2. Pop M, Walker AW, Paulson J, Lindsay B, Antonio M, Hossain MA, Oundo J, Tamboura B, Mai V, Astrovskaya I, Corrada Bravo H, Rance R, Stares M, Levine MM, Panchalingam S, Kotloff K, Ikumapayi UN, Ebruke C, Adeyemi M, Ahmed D, Ahmed F, Alam MT, Amin R, Siddiqui S, Ochieng JB, Ouma E, Juma J, Mailu E, Omore R, Morris JG, Breiman RF, Saha D, Parkhill J, Nataro JP, Stine OC. Diarrhea in young children from low-income countries leads to large-scale alterations in intestinal microbiota composition. Genome Biol. 2014;15(6):1-12. https://doi.org/10.1186/ gb-2014-15-6-r76.

3. Zeller G, Tap J, Voigt AY, Sunagawa S, Kultima JR, Costea PI, Amiot A, Böhm J, Brunetti F, Habermann N, Hercog R, Koch M, Luciani A, Mende DR, Schneider MA, Schrotz-King P, Tournigand C, Tran Van Nhieu J, Yamada T, Zimmermann J, Benes V, Kloor M, Ulrich CM, von Knebel Doeberitz M, Sobhani I, Bork P. Potential of fecal microbiota for early-stage detection of colorectal cancer. Mol Syst Biol. 2014;10:766. https://doi.org/10.15252/msb. 20145645.

4. Quereda JJ, Dussurget O, Nahori M-A, Ghozlane A, Volant S, Dillies M-A, Regnault B, Kennedy S, Mondot S, Villoing B, Cossart P, Pizarro-Cerda J. Proc Natl Acad Sci U S A. 2016;113:5706-11. https://doi.org/10.1073/pnas.1523899113.

5. Veiga P, Gallini CA, Beal C, Michaud M, Delaney ML, DuBois A, Khlebnikov A, van Hylckama Vlieg JE, Punit S, Glickman J, et al. Bifidobacterium animalis subsp. lactis fermented milk product reduces inflammation by altering a niche for colitogenic microbes. Proc Natl Acad Sci. 2010;107(42):18132-7. https://doi.org/10.1073/pnas.1011737107.

6. Westcott SL, Schloss PD. De novo clustering methods outperform reference-based methods for assigning $16 \mathrm{~S}$ rRNA gene sequences to operational taxonomic units. PeerJ. 2015;3:1487. https://doi.org/10.7717/peerj.1487.

7. Schloss PD, Westcott SL, Ryabin T, Hall JR, Hartmann M, Hollister EB, Lesniewski RA, Oakley BB, Parks DH, Robinson CJ, Sahl JW, Stres B, Thallinger GG, Van Horn DJ, Weber CF. Introducing mothur: open-source, platform-independent, community-supported software for describing and comparing microbial communities. Appl Environ Microbiol. 2009;75:7537-41. https://doi.org/10.1128/AEM.01541-09.

8. Edgar RC. UPARSE: highly accurate OTU sequences from microbial amplicon reads. Nat Methods. 2013;10:996-8. https://doi.org/10.1038/nmeth.2604.

9. Callahan BJ, McMurdie PJ, Rosen MJ, Han AW, Johnson AJA, Holmes SP. DADA2: high-resolution sample inference from Illumina amplicon data. Nat Methods. 2016;13:581-3. https://doi.org/10.1038/nmeth.3869.

10. Rognes T, Flouri T, Nichols B, Quince C, Mahé F. VSEARCH: a versatile open source tool for metagenomics. PeerJ. 2016:4:2584. https://doi.org/10.7717/peerj.2584.

11. Caporaso JG, Kuczynski J, Stombaugh J, Bittinger K, Bushman FD, Costello EK, Fierer N, Pena AG, Goodrich JK, Gordon J, et al. QIIME allows analysis of high-throughput community sequencing data. Nat Methods. 2010;7(5): 335-6. https://doi.org/10.1038/nmeth.f.303.

12. MCDonald D, Clemente JC, Kuczynski J, Rideout JR, Stombaugh J, Wendel D, Wilke A, Huse S, Hufnagle J, Meyer $\mathrm{F}$, et al. The Biological Observation Matrix (BIOM) format or: how I learned to stop worrying and love the ome-ome. GigaScience. 2012;1(1):2047-17X. https://doi.org/10.1186/2047-217X-1-7.

13. Paulson JN, Pop M, Bravo HC. Metastats: an improved statistical method for analysis of metagenomic data. Genome Biol. 2011;12(1):1-27.

14. Paulson JN, Stine OC, Bravo HC, Pop M. Differential abundance analysis for microbial marker-gene surveys. Nat Methods. 2013;10:1200-2. https://doi.org/10.1038/nmeth.2658.

15. Love MI, Huber W, Anders S. Moderated estimation of fold change and dispersion for RNA-seq data with DESeq2. Genome Biol. 2014;15:550. https://doi.org/10.1186/s13059-014-0550-8.

16. Robinson MD, McCarthy DJ, Smyth GK. edgeR: a Bioconductor package for differential expression analysis of digital gene expression data. Bioinformatics. 2010;26(1):139-40. https://doi.org/10.1093/bioinformatics/btp616. 
17. McMurdie PJ, Holmes S. Waste not, want not: why rarefying microbiome data is inadmissible. PLoS Comput Biol. 2014;10(4):1003531. https://doi.org/10.1371/journal.pcbi.1003531.

18. Jonsson V, Österlund T, Nerman O, Kristiansson E. Statistical evaluation of methods for identification of differentially abundant genes in comparative metagenomics. BMC Genomics. 2016;17(1):78. https://doi.org/10. 1186/s12864-016-2386-y.

19. Escudié F, Auer L, Bernard M, Mariadassou M, Cauquil L, Vidal K, Maman S, Hernandez-Raquet G, Combes S, Pascal G. FROGS: find, rapidly, OTUs with galaxy solution. Bioinformatics. 2017;34(8):1287-94. https://doi.org/10. 1093/bioinformatics/btx791.

20. Batut B, Gravouil K, Defois C, Hiltemann S, Brugère J-F, Peyretaillade E, Peyret P. ASaiM: a Galaxy-based framework to analyze microbiota data. GigaScience. 2018;7(6):057.

21. Gonzalez A, Navas-Molina JA, Kosciolek T, McDonald D, Vázquez-Baeza Y, Ackermann G, DeReus J, Janssen S, Swafford AD, Orchanian SB, Sanders JG, Shorenstein J, Holste H, Petrus S, Robbins-Pianka A, Brislawn CJ, Wang M, Rideout JR, Bolyen E, Dillon M, Caporaso JG, Dorrestein PC, Knight R. Qiita: rapid, web-enabled microbiome meta-analysis. Nat Methods. 2018;15:796-8. https://doi.org/10.1038/s41592-018-0141-9.

22. Thang MW, Chua X-Y, Price G, Gorse D, Field MA. MetaDEGalaxy: Galaxy workflow for differential abundance analysis of 16 s metagenomic data. F1000Research. 2019;8:

23. McMurdie PJ, Holmes S. Shiny-phyloseq: Web application for interactive microbiome analysis with provenance tracking. Bioinformatics. 2015;31:282-3. https://doi.org/10.1093/bioinformatics/btu616.

24. Wagner J, Chelaru F, Kancherla J, Paulson JN, Zhang A, Felix V, Mahurkar A, Elmqvist N, Corrada Bravo H. Metaviz: interactive statistical and visual analysis of metagenomic data. Nucleic Acids Res. 2018;46(6):2777-87. https://doi. org/10.1093/nar/gky136.

25. Huse SM, Mark Welch DB, Voorhis A, Shipunova A, Morrison HG, Eren AM, Sogin ML. VAMPS: a website for visualization and analysis of microbial population structures. BMC Bioinformatics. 2014;15:41. https://doi.org/10. 1186/1471-2105-15-41.

26. Afgan E, Baker D, Batut B, van den Beek M, Bouvier D, Cech M, Chilton J, Clements D, Coraor N, Grüning BA, Guerler A, Hillman-Jackson J, Hiltemann S, Jalili V, Rasche H, Soranzo N, Goecks J, Taylor J, Nekrutenko A, Blankenberg D. The Galaxy platform for accessible, reproducible and collaborative biomedical analyses: 2018 update. Nucleic Acids Res. 2018;46:537-44. https://doi.org/10.1093/nar/gky379.

27. Dickson LB, Jiolle D, Minard G, Moltini-Conclois I, Volant S, Ghozlane A, Bouchier C, Ayala D, Paupy C, Moro CV, et al. Carryover effects of larval exposure to different environmental bacteria drive adult trait variation in a mosquito vector. Sci Adv. 2017;3(8):1700585. https://doi.org/10.1126/sciadv.1700585.

28. Langmead B, Trapnell C, Pop M, Salzberg SL. Ultrafast and memory-efficient alignment of short DNA sequences to the human genome. Genome Biol. 2009;10(3):25. https://doi.org/10.1186/gb-2009-10-3-r25.

29. Criscuolo A, Brisse S. AlienTrimmer: a tool to quickly and accurately trim off multiple short contaminant sequences from high-throughput sequencing reads. Genomics. 2013;102(5-6):500-6. https://doi.org/10.1016/j.ygeno.2013.07. 011.

30. Zhang J, Kobert K, Flouri T, Stamatakis A. PEAR: a fast and accurate Illumina Paired-End reAd mergeR. Bioinformatics. 2014;30(5):614-20. https://doi.org/10.1093/bioinformatics/btt593.

31. Bokulich NA, Kaehler BD, Rideout JR, Dillon M, Bolyen E, Knight R, Huttley GA, Caporaso JG. Optimizing taxonomic classification of marker-gene amplicon sequences with QIIME 2's q2-feature-classifier plugin. Microbiome. 2018;6(1):90.

32. Pruesse E, Quast C, Knittel K, Fuchs BM, Ludwig W, Peplies J, Glöckner FO. SILVA: a comprehensive online resource for quality checked and aligned ribosomal RNA sequence data compatible with ARB. Nucleic Acids Res. 2007;35(21): 7188-96. https://doi.org/10.1093/nar/gkm864.

33. DeSantis TZ, Hugenholtz P, Larsen N, Rojas M, Brodie EL, Keller K, Huber T, Dalevi D, Hu P, Andersen GL. Greengenes, a chimera-checked 16S rRNA gene database and workbench compatible with ARB. Appl Environ Microbiol. 2006;72:5069-72. https://doi.org/10.1128/AEM.03006-05.

34. Tang J, Iliev ID, Brown J, Underhill DM, Funari VA. Mycobiome: approaches to analysis of intestinal fungi. J Immunol Methods. 2015;421:112-21. https://doi.org/10.1016/j.jim.2015.04.004.

35. Abarenkov K, Henrik Nilsson R, Larsson K-H, Alexander IJ, Eberhardt U, Erland S, Høiland K, Kjøller R, Larsson E, Pennanen T, Sen R, Taylor AFS, Tedersoo L, Ursing BM, Vrålstad T, Liimatainen K, Peintner U, Köljalg U. The UNITE database for molecular identification of fungi-recent updates and future perspectives. New Phytol. 2010;186:281-5. https://doi.org/10.1111/j.1469-8137.2009.03160.x.

36. Findley K, Oh J, Yang J, Conlan S, Deming C, Meyer JA, Schoenfeld D, Nomicos E, Park M, Sequencing NISCC, Kong HH, Segre JA. Topographic diversity of fungal and bacterial communities in human skin. Nature. 2013;498: 367-70. https://doi.org/10.1038/nature12171

37. Yarza P, Yilmaz P, Pruesse E, Glöckner FO, Ludwig W, Schleifer K-H, Whitman WB, Euzéby J, Amann R, Rosselló-Móra R. Uniting the classification of cultured and uncultured bacteria and archaea using 16S rRNA gene sequences. Nat Rev Microbiol. 2014;12:635-45. https://doi.org/10.1038/nrmicro3330.

38. Wang Q, Garrity GM, Tiedje JM, Cole JR. Naive Bayesian classifier for rapid assignment of rRNA sequences into the new bacterial taxonomy. Appl Environ Microbiol. 2007;73:5261-7. https://doi.org/10.1128/AEM.00062-07.

39. Katoh K, Standley DM. MAFFT multiple sequence alignment software version 7: improvements in performance and usability. Mol Biol Evol. 2013;30(4):772-80. https://doi.org/10.1093/molbev/mst010.

40. Criscuolo A, Gribaldo S. BMGE (Block Mapping and Gathering with Entropy): a new software for selection of phylogenetic informative regions from multiple sequence alignments. BMC Evol Biol. 2010;10(1):210. https://doi. org/10.1186/1471-2148-10-210.

41. Price MN, Dehal PS, Arkin AP. FastTree: computing large minimum evolution trees with profiles instead of a distance matrix. Mol Biol Evol. 2009;26(7):1641-50. https://doi.org/10.1093/molbev/msp077.

42. Lozupone C, Knight R. UniFrac: a new phylogenetic method for comparing microbial communities. Appl Environ Microbiol. 2005;71:8228-35. https://doi.org/10.1128/AEM.71.12.8228-8235.2005. 
43. Sims $D$, Sudbery I, llott NE, Heger A, Ponting CP. Sequencing depth and coverage: key considerations in genomic analyses. Nat Rev Genet. 2014;15:121-32. https://doi.org/10.1038/nrg3642.

44. McMurdie PJ, Holmes S. phyloseq: an R package for reproducible interactive analysis and graphics of microbiome census data. PloS ONE. 2013;8(4):61217. https://doi.org/10.1371/journal.pone.0061217.

45. Evans C, Hardin J, Stoebel DM. Selecting between-sample RNA-Seq normalization methods from the perspective of their assumptions. Brief Bioinform. 2017;19(5):776-92. https://doi.org/10.1093/bib/bbx008.

46. Dillies M-A, Rau A, Aubert J, Hennequet-Antier C, Jeanmougin M, Servant N, Keime C, Marot G, Castel D, Estelle J, et al. A comprehensive evaluation of normalization methods for Illumina high-throughput RNA sequencing data analysis. Brief Bioinform. 2013;14(6):671-83. https://doi.org/10.1093/bib/bbs046.

47. Ondov BD, Bergman NH, Phillippy AM. Interactive metagenomic visualization in a Web browser. BMC Bioinformatics. 2011;12(1):385. https://doi.org/10.1186/1471-2105-12-385.

48. Conway JR, Lex A, Gehlenborg N. UpSetR: an R package for the visualization of intersecting sets and their properties. Bioinformatics. 2017;33(18):2938-40. https://doi.org/10.1093/bioinformatics/btx364.

49. Hourdel V, Volant S, O'Brien DP, Chenal A, Chamot-Rooke J, Dillies M-A, Brier S. MEMHDX: an interactive tool to expedite the statistical validation and visualization of large HDX-MS datasets. Bioinformatics. 2016;32(22):3413-9. https://doi.org/10.1093/bioinformatics/btw420.

50. Vonaesch P, Morien E, Andrianonimiadana L, Sanke H, Mbecko J-R, Huus KE, Naharimanananirina T, Gondje BP, Nigatoloum SN, Vondo SS, et al. Stunted childhood growth is associated with decompartmentalization of the gastrointestinal tract and overgrowth of oropharyngeal taxa. Proc Natl Acad Sci. 2018;115(36):8489-98. https://doi. org/10.1073/pnas.1806573115.

51. Acinas SG, Sarma-Rupavtarm R, Klepac-Ceraj V, Polz MF. PCR-induced sequence artifacts and bias: insights from comparison of two 16S rRNA clone libraries constructed from the same sample. Appl Environ Microbiol. 2005;71(12): 8966-9.

52. Sipos R, Székely AJ, Palatinszky M, Révész S, Márialigeti K, Nikolausz M. Effect of primer mismatch, annealing temperature and PCR cycle number on 16S rRNA gene-targetting bacterial community analysis. FEMS Microbiol Ecol. 2007;60:341-50. https://doi.org/10.1111/j.1574-6941.2007.00283.x.

\section{Publisher's Note}

Springer Nature remains neutral with regard to jurisdictional claims in published maps and institutional affiliations.

- fast, convenient online submission

- thorough peer review by experienced researchers in your field

- rapid publication on acceptance

- support for research data, including large and complex data types

- gold Open Access which fosters wider collaboration and increased citations

- maximum visibility for your research: over $100 \mathrm{M}$ website views per year

At BMC, research is always in progress.

Learn more biomedcentral.com/submissions 